\title{
LA ESTRATEGIA DE SEGURIDAD NACIONAL: LA PERSPECTIVA Y EL ENFOQUE ESTRATÉGICO DE AFRICA SUBSAHARIANA
}

\author{
María-Ángeles Alaminos ${ }^{1}$ \\ Universidad Complutense de Madrid (UCM) / UNISCI
}

\begin{abstract}
Resumen:
La Estrategia de Seguridad Nacional española, aprobada el 30 de mayo de 2013, insiste en un enfoque o "visión integral" de la seguridad nacional. Uno de los aspectos fundamentales de este enfoque integral sería la relación que establece entre la seguridad y el desarrollo, es decir, la interdependencia y la vinculación estrecha entre ambos. En este artículo intentamos esclarecer el enfoque de la Estrategia Nacional de Seguridad hacia África Subsahariana en el marco de este enfoque integral que fusiona las vertientes securitaria y desarrollista. Este nexo seguridad-desarrollo genera una aproximación compleja que puede favorecer la búsqueda de objetivos meramente securitarios, obviando otras dimensiones, como el desarrollo económico, centrando de esta manera el interés en "el fomento de nuestra seguridad", en detrimento de relaciones equilibradas con los terceros países y alejándose del análisis de las necesidades sobre el terreno.
\end{abstract}

Palabras clave: Estrategia de Seguridad Nacional, enfoque integral, seguridad, África Subsahariana, nexo seguridad-desarrollo.

Title in English: "Strategy of National Security: Perspective and Strategic Focus of Sub-Saharian Africa”

\section{Abstract:}

The Spanish National Security Strategy adopted on 30 May 2013 emphasizes a "holistic approach" to national security. One of the key aspects of this integrated approach is the establishment of a close relationship between security and development. In this sense, security and development would be interlinked and mutually reinforcing. Based on this comprehensive approach and the focus provided by the Spanish National Security Strategy and by the European Security Strategy, this article tries to highlight the specific challenges with emphasis on Sub-Saharan Africa. The analysis of the security-development nexus indicates the generation of an approach that proposes and enables the continuous search for purely security objectives, ignoring other aspects such as the economic development of the individual countries. Thus the focus has been on "promoting our security" to the detriment of the development of more equitable and balanced relations with third countries.

Keywords: National Security Strategy, Comprehensive Approach, Security, Sub-Saharan Africa, Security, Development Nexus.

Copyright (C) UNISCI, 2014.

Las opiniones expresadas en estos artículos son propias de sus autores, y no reflejan necesariamente la opinión de UNISCI. The views expressed in these articles are those of the authors, and do not necessarily reflect the views of UNISCI.

\footnotetext{
${ }^{1}$ María Ángeles Alaminos Hervás es becaria de investigación en la Universidad Complutense de Madrid, investigadora junior de UNISCI y miembro del Foro Hispano-Argelino.

Dirección: Departamento de Estudios Internacionales, Facultad de Ciencias Políticas y Sociología, UCM, Campus de Somosaguas, 28223 Madrid, España.

E-mail: $\underline{\text { ma_alaminos_h@hotmail.com. }}$
} 


\section{Introducción}

El progresivo declive de la Unión Europea (UE) como actor con capacidad de influencia internacional ha contribuido a la intensificación del proceso de reflexión en torno a los ámbitos de la política exterior y de seguridad europea. ${ }^{2}$ En este artículo veremos cómo en la Estrategia Europea de Seguridad y en el correspondiente informe sobre su aplicación se identifican determinadas amenazas prioritarias y objetivos estratégicos, aunque los documentos tienen una orientación demasiado general y no especifican aspectos detallados de las líneas de acción concretas a seguir. España, como Estado miembro de la Unión, en su Estrategia de Seguridad Nacional, y de la misma manera en la Estrategia Española de Seguridad que la precede, afirma su vocación europeísta y su compromiso con el enfoque y el avance de la UE.

Se puede observar una convergencia en lo que respecta tanto a la identificación de las amenazas como a los objetivos estratégicos, con algunas diferencias que no alteran la posición de la UE como marco de referencia fundamental para la aproximación de la política exterior de España. En el ámbito geográfico de África Subsahariana, la visión de España y de la UE es similar en cuanto a la percepción del continente y de los desafíos a la seguridad que de él emanan, y como intentaremos reflejar en este artículo, constituye una visión bastante limitada e imbuida de la vinculación entre las dimensiones securitaria y desarrollista, en el seno de un enfoque de carácter liberal, que privilegia la búsqueda de la seguridad, y justifica de esta manera el desarrollo de un tipo de intervencionismo en pos de un proceso de (re)construcción de las estructuras del Estado que garanticen la estabilidad y eviten la diseminación de las amenazas.

Por otro lado, el discurso relativo a África Subsahariana, y la preocupación acerca de los conflictos violentos, el subdesarrollo y la debilidad de las estructuras estatales, no se ve acompañado de una implicación coherente y efectiva de los recursos disponibles, generándose una brecha entre los objetivos ambiciosos en el papel en términos de seguridad, y la limitada atención que se puede o quiere prestar al continente, tanto en la esfera de la seguridad como en el ámbito del desarrollo.

En el presente artículo, abordaremos en primer lugar la Estrategia de Seguridad Nacional en el marco fundamental de referencia que supone la Unión Europea, con el objetivo de establecer ciertas similitudes respecto a los objetivos estratégicos, la definición de las amenazas y la aproximación a los desafíos a la seguridad. En segundo lugar analizaremos la visión que la Estrategia de Seguridad Nacional tiene de África Subsahariana, reflejando los aspectos más significativos de su caracterización del continente africano, como la percepción de las amenazas, y analizaremos cuáles son las consecuencias que trae consigo la asociación entre seguridad y desarrollo. En último lugar, el artículo se cierra con una breve conclusión.

\footnotetext{
${ }^{2}$ Ver en este sentido: Fägersten, Björn; Marrone, Alessandro; Ortega, Martín y Parkes, Roderick: "Towards a European Global Strategy: Securing European Influence in a Changing World", European Global Strategy Project (28 de mayo del 2013), pp.1-22; Lasheras, Borja; Pohlmann, Christoph; Katsioulis, Christos y Liberti, Fabio: "European Union Security and Defence White Paper: A Proposal", Berlin, Friedrich-Ebert-Stiftung (Enero de 2010), en

http://library.fes.de/pdf-files/id/ipa/07075.pdf; Laborie Iglesias, Mario A.: "Una propuesta de Libro Blanco para Europa", Revista Española de Defensa (abril 2010), pp. 46-51.
} 


\section{La inserción de la Estrategia de Seguridad Nacional en el marco europeo: Relación y convergencia con la dimensión exterior de la seguridad de la Unión Europea}

En este epígrafe abordaremos la relación de la Estrategia de Seguridad Nacional con la Estrategia Europea de Seguridad ("Una Europa segura en un mundo mejor"), que fue adoptada por la Unión Europea en diciembre de 2003, y que representa, junto con el informe sobre su aplicación de diciembre de 2008 ("Ofrecer seguridad en un mundo en evolución") la dimensión exterior de la seguridad de Europa.

Analizaremos los aspectos principales de ambas estrategias que constituyen elementos comunes y muestran una convergencia tanto de enfoque como de visión de la seguridad. De esta forma intentaremos reflejar, por un lado, la función del marco europeo como referencia fundamental para España en materia de seguridad exterior; por otro lado, las limitaciones o deficiencias de los enfoques elaborados en España y en Europa, enunciando de esta manera algunos de los argumentos que desarrollaremos más extensamente en los siguientes apartados de este artículo.

Este análisis no abarca la dimensión interior de la seguridad, la cual complementa la dimensión exterior de la misma. Compararemos la Estrategia de Seguridad Nacional de 2013 con la Estrategia Europea de Seguridad de 2003, observando a grandes rasgos los elementos comunes y dispares entre ambas, sin tener en consideración la Estrategia de Seguridad Interior de la Unión Europea ("Hacia un modelo europeo de seguridad") que fue aprobada por el Consejo Europeo en marzo de 2010 y cuyos sendos informes sobre su aplicación se elaboraron en noviembre de 2011 y en abril de $2013 .^{3}$

\subsection{Amenazas prioritarias y objetivos estratégicos}

La Unión Europea supone para la política exterior española uno de sus marcos de referencia fundamentales, llegándose a afirmar que "los intereses de España estarán mejor protegidos si la UE se refuerza en el plano interno y en su dimensión exterior". ${ }^{4}$ Por ende, la convergencia de la Estrategia de Seguridad Nacional de España y la Estrategia Europea de Seguridad es a priori una condición altamente esperable, en términos de alineación de los objetivos estratégicos y de similitud en la identificación de las principales amenazas a la seguridad.

La Estrategia Europea de Seguridad es el documento que especifica de forma directa el conjunto de amenazas y desafíos a la seguridad que la UE considera prioritarios. Las principales amenazas que identifica la estrategia europea son el terrorismo, la proliferación de armas de destrucción masiva, los conflictos regionales, la descomposición del Estado y la delincuencia organizada. En el informe de revisión publicado cinco años después a la formulación de la Estrategia, la UE añade los retos de la ciberseguridad, la seguridad energética y el cambio climático. Asimismo, sitúa la proliferación de armas de destrucción

\footnotetext{
${ }^{3}$ La Estrategia de Seguridad Interior de la UE: Hacia un modelo europeo de seguridad, remite de forma sintética a "los principales riesgos y amenazas para la seguridad a los que se enfrenta hoy Europa, como el terrorismo, la delincuencia organizada y grave, el tráfico de drogas, la ciberdelincuencia, la trata de seres humanos, la explotación sexual de menores y la pornografía infantil, la delincuencia económica y la corrupción, el tráfico de armas y la delincuencia transfronteriza, se adaptan muy rápidamente a la evolución científica y tecnológica, en su intento de aprovecharse ilegalmente y socavar los valores y la prosperidad de nuestras sociedades abiertas", p. 7.

4 "Estrategia de Seguridad Nacional" (2013), p. 13.
} 
masiva, el terrorismo y la delincuencia organizada en primera línea de interés, considerando necesaria una mejor conciliación de las dimensiones exterior e interior de la seguridad.

Si comparamos la identificación de las amenazas en las dos estrategias, podemos observar que ambas comparten la enumeración de los retos a la seguridad que se efectúa en la estrategia europea. Esos desafíos son comunes, como no podía ser de otra manera, y la estrategia española añade además, otros riesgos y amenazas adicionales fruto del contexto político actual, como son la inestabilidad económica y financiera, los flujos migratorios irregulares, el espionaje, las emergencias y catástrofes, la vulnerabilidad del espacio marítimo y la vulnerabilidad de las infraestructuras críticas y servicios esenciales. ${ }^{5}$

Al igual que en la Estrategia de Seguridad Nacional de 2013, donde África Occidental ocupa el centro del interés español en el continente africano, en la Estrategia Europea de Seguridad la región se manifiesta también como una zona de especial preocupación, en particular por la presencia de conflictos violentos sin resolver y por la amenaza a la seguridad que suponen los denominados Estados fallidos o frágiles: "Si se los descuida, los Estados en descomposición y la delincuencia organizada se extienden, como hemos podido ver en África occidental". ${ }^{6}$ De forma más general, África Subsahariana es caracterizada en el texto como un continente pobre, donde "en muchos casos, el fracaso económico está ligado a problemas políticos y a conflictos violentos".

De la identificación de las amenazas prioritarias para la seguridad europea deriva la formulación de los objetivos estratégicos, que se centran principalmente en hacer frente a las amenazas enumeradas anteriormente, así como en generar seguridad en los países vecinos para de esta forma garantizar la seguridad de la UE, y en fomentar la existencia de un "sistema multilateral eficaz" 8 para hacer frente a las amenazas a la seguridad internacional.

No obstante, el compromiso con un orden internacional basado en el Derecho no responde únicamente a la vocación normativa de la UE, y a la implementación de sus principios y valores, sino que obedece también a los intereses europeos en términos de estabilidad, que influyen a la hora de contribuir en el desarrollo de la gobernanza global y de las organizaciones regionales e internacionales. ${ }^{9}$

\subsection{España y la UE, una visión compartida de la seguridad: el nexo seguridad- desarrollo}

Si bien podemos afirmar que tanto la Estrategia Europea de Seguridad como la Estrategia de Seguridad Nacional española comparten un análisis similar en la valoración de los retos a la seguridad y de las amenazas fundamentales, el aspecto más significativo que nos interesa reflejar en este artículo es que ambas estrategias comparten, en esencia, la misma visión subyacente de la seguridad, el mismo enfoque que vincula indisolublemente seguridad y desarrollo.

\footnotetext{
${ }^{5}$ Puede consultarse una tabla comparativa de riesgos y amenazas de la Estrategia Europea y la Estrategia Española de Seguridad (el documento de 2011, no el de 2013, aunque no hay grandes diferencias entre ambos y se caracterizan por la continuidad) en: Instituto español de Estudios Estratégicos: "Análisis comparativo de la Estrategia Española de Seguridad 'Una responsabilidad de todos"', Instituto Español de Estudios Estratégicos (IEEE), Documento de Análisis, nº 17 (26 de junio del 2011).

6 "Estrategia Europea de Seguridad" (2003), p. 7.

${ }^{7}$ Ibid., p. 2.

${ }^{8}$ Ibid., p. 9.

${ }^{9}$ Keohane, Daniel: "Strategic priorities for EU defence policy", FRIDE, Policy Brief, no 146 (February 2013), p. 2 .
} 
"La seguridad es una condición para el desarrollo. El conflicto no sólo destruye las infraestructuras, incluidas las sociales, sino que también fomenta la delincuencia, disuade a los inversores e imposibilita la actividad económica normal. Varios países y regiones han quedado atrapados en un ciclo de conflicto, inseguridad y pobreza". ${ }^{10}$

Este nexo seguridad-desarrollo, el enfoque de la paz liberal y las intervenciones del nuevo humanitarismo, responden a una lógica realista de las relaciones internacionales, en la que el Norte global prioriza sus preocupaciones e intereses securitarios y ve las situaciones en el Sur global como amenazas a las que hay que hacer frente para evitar sus efectos perniciosos. ${ }^{11} \mathrm{Ya}$ desde mediados de la década de los años noventa, los países del norte representaron las nuevas crisis del sur no como desastres humanitarios únicamente, sino como amenazas de carácter político y estratégico que podrían afectar a la seguridad internacional. ${ }^{12} \mathrm{O}$, en otras palabras, "unremediated butchery in foreign lands adversely affects the interests of people at home". ${ }^{13}$

"Incluso en una era de globalización, la geografía sigue siendo importante. A Europa le conviene que los países limítrofes estén bien gobernados. Los vecinos inmersos en conflictos violentos, los Estados débiles en los que prolifera la delincuencia organizada, las sociedades disfuncionales o las explosiones demográficas en nuestras fronteras plantean problemas a Europa". ${ }^{14}$

El fenómeno de las denominadas 'nuevas guerras' ha sido escrutado desde distintos ángulos de análisis por distintos autores. ${ }^{15}$ Además de la interpretación que pone el énfasis en el sentimiento identitario como factor principal para el conflicto ("conflictos interétnicos"), en la Estrategia Europea de Seguridad está presente otro enfoque que privilegia los problemas de gobernanza y capacidad institucional presentes en la mayoría de los países que sufren la violencia de las nuevas guerras, generando Estados fallidos o incluso la desintegración de las estructuras de gobierno, cuando considera que "la mejor protección para nuestra seguridad es un mundo de Estados democráticos bien gobernados". ${ }^{16}$ De la misma manera, en el caso español se afirma en el mismo sentido que "África presenta problemas de estabilidad y conflictos no resueltos, que llegan en ocasiones a amenazar la propia existencia de algunos Estados". 17

El epicentro del interés se sitúa en el Estado, y en consecuencia, las intervenciones en África Subsahariana van a tener como objetivo fundamental el proceso de construcción del

\footnotetext{
10 "Estrategia Europea de Seguridad" (2003), p. 2.

${ }^{11}$ Marriage, Zoë: "Compliance Versus the Ragged Threat: Problem-Solving Security in the Democratic Republic of Congo", Civil Wars, vol. 15, n¹ (Marzo 2013), pp. 19-20.

12 Ver: Dannreuther, Roland (2007): International Security: The Contemporary Agenda, Cambridge, Polity Press, p. 153.

${ }^{13}$ Farer, T.J.: "Humanitarian Intervention Before and After 9/11: Legality and Legitimacy", pp. 53-89, en Holzgrefe, J. L., y Keohane, R. O. (eds.) (2003): Humanitarian Intervention: Ethical, Legal and Political Dilemmas, Cambridge, Cambridge University Press, p.59.

14 "Estrategia Europea de Seguridad" (2003), p. 7.

${ }^{15}$ Véase una síntesis de las principales interpretaciones en Dannreuther (2007), op. cit., pp. 122-123.

16 "Estrategia Europea de Seguridad" (2003), p. 10.

17 "Estrategia de Seguridad Nacional" (2013), p. 17.
} 
Estado ('statebuilding') para generar condiciones de estabilidad que eviten la propagación de las amenazas citadas anteriormente y que permitan el desarrollo en una fase posterior. El objetivo fundamental no es por tanto el desarrollo económico en primer lugar, sino la consecución de una situación de seguridad y estabilidad. Como se considera que algunos Estados en el continente africano no protegen a la población ni proveen los servicios básicos necesarios, sino que constituyen en algunos casos la amenaza para su propia población, esto favorece la justificación para que la comunidad internacional se erija en protector o proveedor de esas poblaciones, favoreciendo un enfoque que enfatiza la necesidad de intervenir, aunque ello puede conllevar en muchas ocasiones consecuencias más negativas que positivas. ${ }^{18}$

\section{El lugar de África Subsahariana en la Estrategia de Seguridad Nacional: Una visión limitada}

"África, clave para la seguridad española", decía la Estrategia Española de Seguridad de 2011; "España tiene intereses particulares en el continente africano", se afirma en la Estrategia de Seguridad Nacional de 2013, en lo que parece un descenso cualitativo en la importancia estratégica y en el interés por el continente africano al sur del Sáhara. En este artículo focalizamos la atención en África Subsahariana exclusivamente debido al hecho de que cuando ambas estrategias $(2011,2013)$ se refieren a 'África', esta denominación remite a África Subsahariana, quedando los países del norte de África englobados en el epígrafe relativo al Mediterráneo (2013) o 'nuestra vecindad del Sur' (2011).

Tanto en 2011 como en 2013, África no ocupa más de una página dentro del documento estratégico, lo cual puede parecer a priori una muestra de escasa atención hacia el continente, o de falta de relevancia estratégica. Sin embargo, cuando lo comparamos con la presencia en el papel del resto de las regiones geográficas, observamos que la parquedad en palabras es similar.

Si bien la Estrategia de Seguridad Nacional constituye "la articulación fundamental de la Seguridad Nacional como Política de Estado"19, y uno de los aspectos principales de cualquier estrategia es la definición y la puesta en funcionamiento de los mecanismos de aplicación necesarios para la realización práctica de tal estrategia, en este artículo no abordaremos la fase de implementación, por considerar que no disponemos de los elementos suficientes para evaluar la fase de aplicación práctica de la estrategia, fundamentalmente por motivos de retraso y demora en la implementación de la estrategia de seguridad por parte de los sucesivos gobiernos en el poder. ${ }^{20}$

\footnotetext{
${ }^{18}$ Nos referimos a que "el énfasis en la seguridad y la restauración de las estructuras militares y de seguridad no garantizan per se ni el desarrollo ni los derechos humanos, la igualdad de género, etc., sino que por el contrario puede ser claramente contraproducente", ver en: Ruiz-Giménez Arrieta, Itziar (con la colaboración de Lourdes Benavides de la Vega): "La política exterior española de construcción de la paz hacia África Subsahariana. Balance de las últimas décadas desde la perspectiva del principio de coherencias políticas", Informe a cargo del Grupo de Estudios Africanos (Noviembre 2011), p.11.

19 "Estrategia de Seguridad Nacional" (2013), p. 8.

${ }^{20}$ A este respecto véase el comentario de Arteaga, que explica cómo la Estrategia Española de Seguridad de 2011 no fue puesta en marcha por falta de tiempo, y cómo la Estrategia de Seguridad Nacional de 2013 va por el mismo camino: "Mucha tarea para el poco tiempo que se da el Gobierno para realizarla: seis meses, teniendo en cuenta que ya ha transcurrido más de un tercio de la legislatura y que el consenso entre los dos grandes partidos se reducirá a medida que avancen las citas electorales", ver en: Arteaga, Félix: "La Estrategia de Seguridad Nacional 2013”, Comentario Elcano, no 37 (31 de mayo de 2013), p.2.
} 
En consecuencia, abordaremos la estrategia desde una óptica conceptual, analizando los aspectos más significativos del contenido y discurso de la misma, los cuales nos pueden ayudar a comprender las características de la visión subyacente de la seguridad y el lugar concreto que ocupa África Subsahariana en el contexto de seguridad español.

Como premisa previa, debemos partir de la base de que ambas estrategias españolas comparten un enfoque común y presentan un alto grado de similitud y continuidad. De hecho el documento de 2013 es muy parecido al de 2011, el contenido es similar, en ocasiones de forma literal o ligeramente modificado, y los aspectos fundamentales de la estrategia son prácticamente iguales, tanto en su concepción general como en el caso particular que nos ocupa (África Subsahariana), lo cual muestra el consenso en materia de seguridad entre los dos partidos políticos que se han sucedido en el gobierno.

Analizaremos ahora los elementos principales respecto a África Subsahariana que hemos identificado en las dos estrategias de seguridad, con el objetivo de evidenciar algunas características significativas de la aproximación española al continente y de la visión que subyace a la definición de las políticas hacia el mismo.

\subsection{Identificación de riesgos y amenazas provenientes de África en la Estrategia de Seguridad Nacional}

Tanto en 2011 como en 2013, la Estrategia define tres zonas "vitales" en África "para los intereses españoles": el Sahel, el Cuerno de África y el Golfo de Guinea. No se identifican únicamente las mismas zonas prioritarias, sino también las mismas amenazas en proveniencia de dichas zonas:

"En las dos primeras confluyen tráficos ilícitos, conflictos étnicos, terrorismo, Estados fallidos, subdesarrollo y extremismos ideológicos (...) En lo que respecta al Golfo de Guinea, la falta de estabilidad en la zona puede generar también inseguridad marítima, muy negativa para el abastecimiento energético y los flujos comerciales". ${ }^{21}$

La Estrategia de Seguridad parece de esta manera reproducir una visión del mundo, común desde el final de la Guerra Fría, según un eje norte-sur, que contribuye a una representación de dos mundos en oposición. Por un lado, un norte próspero, industrializado y en paz. Por otro lado, un sur (donde se inserta África Subsahariana) pobre, donde florece el conflicto, la violencia y el subdesarrollo.

En las dos estrategias de 2011 y 2013 se consideran los conflictos armados como una de las amenazas capitales a la seguridad, y se especifica que en la actualidad la mayor parte de las veces la violencia se desarrolla en forma de conflictos internos / intraestatales, denominación poco desarrollada en el texto, que obvia la extremada importancia de la dimensión transnacional de dichos conflictos.

\footnotetext{
21 "Estrategia de Seguridad Nacional" (2013), pp. 17-18; La Estrategia Española de Seguridad de 2011 se refiere a las mismas amenazas con las mismas palabras: "En las dos primeras, confluyen hechos problemáticos y graves como tráficos ilícitos, conflictos étnicos, terrorismo, Estados fallidos y subdesarrollo (...) La falta de estabilidad política de los Estados en el Golfo de Guinea puede generar también una inseguridad marítima y un incipiente bandidaje, ambos muy negativos para el abastecimiento energético y los flujos comerciales" (p.30).
} 
"Los conflictos armados siguen representando una amenaza capital para la seguridad, aun cuando la interdependencia global ha influido en su morfología y disminuido la probabilidad de confrontaciones clásicas entre Estados. La mayoría de los conflictos de carácter violento son de naturaleza interna". ${ }^{22}$

Además, la naturaleza del conflicto en África lleva asociada en la Estrategia de 2011 y de 2013 la etiqueta de "conflicto étnico". De hecho, los denominados "nuevos conflictos" o "nuevas guerras" se han interpretado frecuentemente como guiados por un sentimiento identitario, ya sea de grupo nacional, étnico o religioso, y por considerar la desaparición del factor ideológico como impulso para el conflicto, a diferencia de lo que ocurría durante la Guerra Fría. ${ }^{23}$

Esto ha generado una visión del Sur como territorio de la violencia causada por las diferencias identitarias, donde las visiones particularistas se oponen a la situación en el Norte, pacífico, democrático y cosmopolita (incluyente). ${ }^{24}$

Esta caracterización presenta limitaciones que han sido puestas en evidencia por diversos autores, ya que representan una visión simplificadora que ha generado una visión disociada del escenario internacional, donde el Sur global presenta desafíos de seguridad ingentes, y el Norte (Occidente), con su visión cosmopolita, actúa en el Sur global para promover su enfoque basado en la paz, la democracia y los valores liberales. De aquí surge el enfoque de la paz liberal y la persecución de esos valores y principios a través de la intervención en el Sur.

Hay muchos autores que ponen en duda la novedad de estos tipos de violencia organizada. Kalyvas defiende que la caracterización de las 'nuevas guerras' como "criminales, despolitizadas, privadas y depredadoras" en contraste con las 'viejas guerras' representadas como "ideológicas, políticas, colectivas e incluso nobles" no es una distinción que sirva a objetivos meramente clasificatorios o de comprensión académica, sino que responde a criterios políticos y motiva respuestas políticas específicas que pueden incluir la intervención humanitaria. $^{25}$

No obstante, aunque las viejas guerras no son tan diferentes de las nuevas guerras, y se han exagerado el carácter criminal y la violencia en detrimento de los aspectos políticos, el cambio de naturaleza de los conflictos contemporáneos y de los significados y significaciones asociados al término 'seguridad' son un hecho palpable tanto en la literatura académica como en la realidad social. ${ }^{26}$

\footnotetext{
22 "Estrategia de Seguridad Nacional" (2013), p. 24. El documento de 2011 prefiere denominarlos conflictos "intraestatales" (p. 43).

${ }^{23}$ Véase el análisis crítico a este respecto de Dannreuther, op.cit., p. 123.

${ }^{24}$ Ver: Kaldor, Mary (2006/1999): New \& Old Wars, $2^{\mathrm{a}}$ ed., Cambridge, Polity, p. 21.

${ }^{25}$ Ver: Kalyvas, Stathis N.: ““New” and "Old” Civil Wars: A Valid Distinction?”, World Politics, vol. 54, n¹ (2001), pp. 99-118.

26 "Les critères mis en avant par les adeptes des "nouvelles guerres" sont éminemment contestables car déjà bien établis dans les “anciennes”, mais cela n'implique pas que rien n'ait changé et que les modalités de la guerre aujourd'hui soient les mêmes qu'avant la fin de la Guerre froide et/ou l'avènement de la globalisation"; Marchal, Roland : "Conclusion”, en Hassner, Pierre y Marchal, Roland (eds.) (2003): Guerres et sociétés: État et violence après la Guerre froide, Paris, Karthala, pp. 571-608 (p. 585).
} 
De hecho, la misma desaparición de la prevalencia de guerra interestatal entre los principales polos de poder ha sido un factor facilitador de la ampliación de la agenda de seguridad, que ha incorporado, en ausencia de su tradicional foco de atención, otros asuntos como el 'medio ambiente, los recursos y la migración'. ${ }^{27}$ Estos tres asuntos se encuentran presentes y entrelazados en el documento estratégico de 2013 al referirse al continente africano como:

"el continente más vulnerable a las consecuencias perversas del cambio climático. Factores medioambientales están ya provocando desplazamientos masivos de población y conflictos asociados principalmente a la escasez del agua y la explotación de los recursos". ${ }^{28}$

\subsection{La asociación entre seguridad y desarrollo}

La división u oposición norte-sur que hemos visto más arriba trae consigo una consecuencia fundamental para nuestro análisis, la asociación o vinculación entre la seguridad y el desarrollo en la agenda de seguridad española hacia África, o bien, la percepción del subdesarrollo del sur como la fuente de amenazas a la seguridad, tanto para el sur como para la seguridad internacional en general. ${ }^{29}$

"Con el objetivo de promover el desarrollo en África e incrementar de esta manera la seguridad en dicho continente y en su entorno, España ha impulsado importantes estrategias bilaterales y multilaterales sociales, económicas e institucionales". 30

El mundo queda así dividido en dos, el mundo desarrollado y el mundo subdesarrollado, dentro del cual se dan las circunstancias que generan amenazas que afectan tanto al Sur como al Norte, porque una de las características de los nuevos conflictos es que no conocen de fronteras, así que se expanden y pueden llegar a penetrar y afectar la seguridad e intereses de Occidente.

"La piratería en el Océano Indico es claro ejemplo de cómo la debilidad de un Estado, Somalia, puede crear una inseguridad que afecta a los intereses de la comunidad internacional y, particularmente, a los intereses de los españoles". ${ }^{31}$

El mundo subdesarrollado de la inseguridad puede afectar al mundo desarrollado seguro. Una vez establecida esta politización y securitización del desarrollo ("la fusión del desarrollo y la seguridad"), los países de occidente se otorgan la misión de gestionar la inseguridad en el Sur

\footnotetext{
${ }^{27}$ Ver: Dannreuther, op. cit, p. 121.

28 "Estrategia de Seguridad Nacional" (2013). En la estrategia de 2011 la referencia es más corta y directa, refiriéndose a África como el continente "en el que tienen lugar, con especial intensidad, fenómenos tan preocupantes como las migraciones descontroladas o el cambio climático".

${ }^{29}$ Ver el análisis de Duffield, Mark (2001): Global Governance and the New Wars: The Merging of Development and Security, London, Zed Books.

30 "Estrategia Española de Seguridad" (2011), p. 30.

31 "Estrategia de Seguridad Nacional" (2013), p. 18.
} 
a través de intervenciones que incluyen la gestión de conflictos y la intervención humanitaria, bajo la égida de una construcción y mantenimiento de la paz de carácter liberal. ${ }^{32}$

\begin{abstract}
"El empleo de nuestras Fuerzas Armadas puede resultar esencial en los conflictos que eventualmente llegaran a desarrollarse tanto dentro como más allá de nuestras fronteras". ${ }^{33}$
\end{abstract}

"La cooperación española, mantiene entre sus objetivos la prevención de conflictos y el regreso y consolidación de las condiciones de paz, seguridad, justicia y equidad en el marco de la legalidad internacional". ${ }^{34}$

De esta manera, la violencia desatada en las guerras civiles del sur desemboca en el norte en el auge de las intervenciones internacionales para terminar los conflictos y en la invocación de una responsabilidad u obligación ante la tragedia humana de la población que sufre los conflictos. Es decir, que acudimos a un "revival of liberal internationalism" y a la emergencia de una norma de intervención humanitaria. ${ }^{35}$

El auge de las intervenciones humanitarias desde principios de los años 90 ha reforzado la visión y el vínculo entre seguridad y desarrollo. Con el fin de la Guerra Fría, la Organización de las Naciones Unidas llevó a cabo un despliegue de operaciones en el sur global, intentando hacer frente a las nuevas situaciones generadas por la naturaleza cambiante de las guerras, cuyas consecuencias cruentas y violaciones de los derechos humanos han servido de justificación para el despliegue de las operaciones humanitarias.

\begin{abstract}
"España debe tener la capacidad de participar en crisis o conflictos por su calidad de socio solidario de las organizaciones internacionales a las que pertenece, así como en operaciones de mantenimiento de la paz, de protección de civiles u otras que afecten a nuestros valores compartidos y se deriven de compromisos adquiridos en organizaciones internacionales como la ONU". ${ }^{36}$
\end{abstract}

Por otro lado, otra característica fundamental es que las políticas de desarrollo y seguridad se construyen desde un discurso impregnado de intereses compartidos y preocupaciones comunes, es decir, propugnan "the assertion of a commonality of interests", en palabras de Marriage. ${ }^{37}$ En la estrategia podemos ver un ejemplo concreto respecto a África en la siguiente cita, en la que se insiste en el reforzamiento (bueno para ellos-bueno para nosotros) de la seguridad tanto en el sur como en el norte:

"La actuación en Malí, en la que se conjuga el apoyo material a sus fuerzas armadas en la lucha contra el terrorismo, la cooperación para reforzar sus fuerzas de seguridad y la

\footnotetext{
${ }^{32}$ Duffield (2001), op. cit.

33 "Estrategia de Seguridad Nacional" (2013), p. 24.

34 "Plan Director de la Cooperación Española 2013-2016", p. 29

${ }^{35}$ Véase Dannreuther, op. cit. , p. 121.

36 "Estrategia de Seguridad Nacional" (2013), p. 25.

37 Marriage se refiere a "assertion of a commonality of interests" en estrecha vinculación con "neoliberal notions of security based on consensus"; Los donantes buscan el consenso con el Sur a la hora de promover sus políticas securitarias y de buen gobierno, aunque esta aproximación consensual puede desaparecer cuando los gobiernos del Sur no responden a las demandas del Norte de la forma deseada y voluntariamente; Marriage, op. cit., pp. 19 y ss.
} 
ayuda para fortalecer las estructuras del Estado y las capacidades de la sociedad civil, muestra las amplias posibilidades de cooperación con estos países, para reforzar su seguridad y la nuestra". ${ }^{38}$

\subsection{La brecha entre el discurso y la realidad}

La asociación conceptual entre seguridad y desarrollo es evidente en los documentos estratégicos de 2011 y 2013, lo cual refleja una visión de la seguridad compleja y con elementos polémicos, discutida más arriba. Sin embargo, la brecha entre el discurso y la realidad se manifiesta rápidamente al comparar por ejemplo lo que se dice en la estrategia de seguridad de 2013 con el contenido del correspondiente Plan director de la cooperación española para el periodo 2013-2016.

La distancia existente entre el discurso y la realidad no es de por sí algo sorprendente cuando nos referimos a determinados aspectos de la acción exterior, aunque sí llama la atención constatar las contradicciones existentes entre los documentos relativos a la seguridad y al desarrollo antes incluso de su implementación y de la activación de los mecanismos necesarios para su realización efectiva. Las diferencias entre el enfoque relativo a África Subsahariana en la Estrategia de Seguridad Nacional y en el Plan Director de la Cooperación Española nos permiten identificar algunos gaps significativos y contradicen en parte, al menos en el ámbito geográfico que nos ocupa, la visión del enfoque integral de la seguridad y el principio de unidad de acción. ${ }^{39}$ En el Plan Director se insiste también, en convergencia con la Estrategia de Seguridad, en la contribución a "una acción exterior más integral".

Lo que no está claro es cómo se va a hacer efectivo el enfoque integral y cómo va a elaborarse la siguiente afirmación: "España tiene intereses particulares en el continente africano, que se han de articular a través de relaciones de cooperación" ${ }^{40}$, cuando se adopta simultáneamente una importante disminución presupuestaria y se establece que no es posible "mantener ciertos compromisos internacionales, como el adquirido en el seno de la UE para alcanzar el 0'56\% de AOD/RNB (objetivo establecido para 2010), o la meta largamente reivindicada del 0'7\% AOD/RNB". ${ }^{41}$

En efecto, en el medio plazo la cooperación española concentrará (limitará) su atención en un conjunto de 23 países de asociación, de los cuales únicamente seis se encuentran en África Subsahariana: tres en África Subsahariana Occidental, que disfruta de 'especial atención' (Mali, Níger y Senegal), y otros tres países en África Oriental, Central y Austral (Etiopía, Guinea Ecuatorial y Mozambique). ${ }^{42}$

Esta es la contradicción más flagrante del plan director de la cooperación con la estrategia de seguridad, la cual define tres zonas "vitales" para la seguridad y los intereses de

\footnotetext{
38 "Estrategia Nacional de Seguridad" (2013), p. 17.

${ }^{39}$ La unidad de acción es uno de los principios informadores de la Estrategia de Seguridad Nacional, "que supone la implicación, la coordinación y la armonización de todos los actores y los recursos del Estado bajo la dirección del Presidente del Gobierno, así como la colaboración público-privada y la implicación de la sociedad en general. El enfoque integral de la seguridad, comprensivo de todas sus dimensiones, justifica este principio de acción y precisa, a su vez, de una gestión completa a través de un Sistema de Seguridad Nacional impulsado y liderado por el Presidente del Gobierno", (p. 9).

40 "Estrategia de Seguridad Nacional" (2013), p. 17.

41 "Plan Director de la Cooperación Española 2013-2016", p. 75.

${ }^{42}$ Ibid., pp. 37-38.
} 
España en las próximas décadas: el Sahel, el Cuerno de África y el Golfo de Guinea, que se encuentran reducidas a su mínima expresión en el ámbito de la cooperación.

La importancia otorgada (en el papel) a África en el plano estratégico, el énfasis en la prevención y la gestión de conflictos, la debilidad de los Estados y la vinculación entre la seguridad y el desarrollo, según la cual "la Cooperación Española trabajará por potenciar las relaciones con otros países en las que confluyan los intereses estratégicos de España con las responsabilidades globales" ${ }^{43}$ no parecen verse correspondidos con la medida tomada por la cooperación española de cerrar o rediseñar nueve de los programas país existentes en África Subsahariana: Angola, Cabo Verde, Gambia, Guinea, Guinea-Bissau, Namibia, República Democrática del Congo, Sudán y Sudán del Sur. Podemos afirmar que los desafíos a la seguridad enunciados en la estrategia de 2013, y las tres zonas geográficas identificadas en África Subsahariana, no se corresponden con el enfoque reductor y limitado adoptado en el plan director de la cooperación.

Este aspecto puede ser una señal del lugar secundario que ocupa África en la identificación de los problemas de seguridad por parte de España, o también el reflejo de la falta de implicación o interés ante algunos de los conflictos y problemas a la estabilidad más importantes, de mayor duración y con mayor número de víctimas en África, como muestra la exclusión de Sudán, Sudán del Sur y República Democrática del Congo de los futuros programas de desarrollo.

En este sentido, encontramos una mayor convergencia entre el documento de la Estrategia española de seguridad de 2011 y el Plan Director de la cooperación española para el periodo 2009-2012 que en el caso de los respectivos documentos de 2013. El Plan Director 2009-2012 consideraba África Subsahariana como una prioridad para el desarrollo: "el despliegue en el África Subsahariana será acorde con la prioridad que tiene esta región en la nueva política española de cooperación al desarrollo" y África Occidental como una "región prioritaria para la Cooperación Española". 44 Asimismo, tanto en el documento del Plan África 2006-2008 como en el segundo Plan África para el periodo 2009-2012, se manifestaba la misma relevancia del continente africano para la política exterior española. ${ }^{45}$

Podemos concluir esta sección afirmando que existe una mayor continuidad en el ámbito de la seguridad que en la esfera del desarrollo. Las estrategias de 2013 y de 2011 son muy parecidas, no presentan diferencias fundamentales, representan una continuidad y articulan una política de Estado. Los planes directores de desarrollo sin embargo presentan muchas diferencias y se ven muy afectados por el contexto económico, hasta el punto de hacer desaparecer en el plan actual los programas para un número importante de países que deberían ser prioritarios desde el punto de vista de los desafíos que plantean, contradiciendo de esta forma la atención que se les dedica en el texto de la estrategia de seguridad vigente.

\footnotetext{
${ }^{43}$ Ibid., p. 16.

44 "Estrategia Española de Seguridad" (2011), pp. 88 y 212; otras referencias a la consideración de África Subsahariana como prioridad pueden verse en las páginas 72 y 212.

${ }^{45}$ Véase un desarrollo más extenso sobre en este sentido en Alaminos Hervás, María Ángeles: "La política exterior de España hacia África Subsahariana a través del análisis crítico de los Planes África", UNISCI Discussion Papers, nº27 (Octubre 2011), pp. 189-197, en

http://revistas.ucm.es/index.php/UNIS/article/view/38149/36901.
} 


\section{Conclusión}

La Estrategia de Seguridad Nacional aprobada el 30 de mayo de 2013 insiste en un enfoque o "visión integral" de la seguridad nacional. Esta visión responde a la necesidad de adaptarse a "las transformaciones globales" y de dar respuesta "a los crecientes desafíos" del mundo actual, mediante un concepto de seguridad que se define como:

"amplio y dinámico, para cubrir todos los ámbitos concernientes a la seguridad del Estado y de sus ciudadanos, que son variables según las rápidas evoluciones del entorno estratégico y abarcan desde la defensa del territorio a la estabilidad económica y financiera o la protección de las infraestructuras críticas". ${ }^{46}$

En la Estrategia Española de Seguridad aprobada en junio de 2011 ya se hacía hincapié en el concepto de "enfoque integral", concepto básico que la estrategia pretendía "impulsar" y que se definía como la integración de "todas y cada una de las dimensiones de la seguridad, haciéndolas converger hacia objetivos comunes y conscientes de las múltiples relaciones que existen entre ellas". 47

Es además un concepto que remite al "enfoque multidimensional" de Naciones Unidas, y al "comprehensive approach" o enfoque integral de la Unión Europea (UE), organizaciones que han desarrollado progresivamente en las últimas décadas un enfoque holístico como guía de su actividad en el ámbito de la seguridad, de forma particular en lo referente a la gestión de crisis y la resolución de conflictos. ${ }^{48}$ La Estrategia se inserta en un marco europeo y multilateral, considerados necesarios e imprescindibles ya que "las respuestas unilaterales y aisladas no son eficaces, por su carácter incompleto y parcial, frente a unos retos que exigen un enfoque multidisciplinar y una acción conjunta". ${ }^{4}$

Sin embargo, el término "enfoque integral" es ambiguo, se define de forma amplia pero inespecífica y genera diversas interpretaciones en función de los objetos que hay que asegurar, del porqué y de la forma para hacerlo, mediante la integración o coordinación de diversas políticas, mecanismos e instrumentos. ${ }^{50}$

Uno de los aspectos fundamentales del enfoque integral es, como hemos visto, la relación que establece entre la seguridad y el desarrollo, es decir, la interdependencia y la vinculación estrecha entre ambos, o bien, la relación entre el subdesarrollo y la inseguridad o el conflicto.

En este artículo hemos intentado esclarecer el enfoque de la Estrategia Nacional de Seguridad hacia África Subsahariana en el marco de este enfoque integral que fusiona las

\footnotetext{
46 "Estrategia de Seguridad Nacional" (2013), pp. 5-6.

47 "Estrategia Española de Seguridad" (2011), p. 16.

${ }^{48}$ Véase la Comunicación Conjunta de la Comisión Europea y la Alta Representante de la UE para Asuntos Exteriores y Política de Seguridad al Parlamento Europeo y al Consejo: "El enfoque integral adoptado por la UE en relación con los conflictos y las crisis exteriores", JOIN (2013) 30 final, Bruselas (11 de diciembre del 2013).

49 "Estrategia Española de Seguridad" (2011), p. 6.

${ }^{50}$ Véase a este respecto por ejemplo el análisis de Drent, Margriet: “The EU's Comprehensive Approach to Security: A Culture of Co-ordination?", Studia Diplomatica, vol. 2, n LXIV (2011); también Woollard, Catherine: "The EU and the Comprehensive Approach", Discussion points for the Civil Society Dialogue Network meeting: The EU's Comprehensive Approach to Exernal Action: Gathering Civil Society Input, Bruselas (January 2013).
} 
vertientes securitaria y desarrollista. Este nexo seguridad-desarrollo genera una aproximación compleja y polémica que puede favorecer la búsqueda de objetivos meramente securitarios, obviando otras dimensiones, como el desarrollo económico ${ }^{51}$, centrando de esta manera el interés en "el fomento de nuestra seguridad" 52 , en detrimento de relaciones equilibradas con los terceros países y alejándose del análisis de las necesidades sobre el terreno.

Además, podemos afirmar para concluir que el lugar que ocupa África Subsahariana en la estrategia de seguridad de España no es un lugar prioritario (así como no lo es tampoco en el caso de la UE). África adolece de la falta de implicación y voluntad política, así como de la falta de dedicación de recursos, generando una contradicción entre el discurso oficial de los documentos relativos a la seguridad y al desarrollo y la práctica, lo cual origina un gap anterior incluso a la activación de los mecanismos necesarios para la implementación de la estrategia.

\footnotetext{
${ }^{51}$ Véase el desarrollo del argumento en Duffield, op. cit.

52 "Estrategia de Seguridad Nacional" (2013), p. 17.
} 